\title{
Duplicidade da artéria renal em cão
}

\author{
Renal artery duplicity in dog
}

Fabrício Singaretti de Oliveira ${ }^{\mathrm{I}}$ Gregório Corrêa Guimarães ${ }^{\mathrm{II}}$

\begin{abstract}
- NOTA -
\section{RESUMO}

Alterações vasculares renais são observadas com baixa freqüência em medicina veterinária e podem ter implicações diretas no funcionamento normal destes órgãos devido à alteração do fluxo sangüíneo. Assim, o objetivo deste relato é descrever um caso de duplicidade da artéria renal em uma cadela de cinco anos, sem raça definida e com $18 \mathrm{~kg}$. $O$ animal veio a óbito após hemoparasitose crônica e foi submetido à injeção de látex pela aorta torácica, na altura do sexto espaço intercostal esquerdo. O animal foi dissecado após fixação com solução de formol a 10\%, no laboratório de Anatomia Animal da Faculdade de Medicina Veterinária da UNICASTELO, Campus Fernandópolis, SP, visando-se à evidenciação dos ramos da aorta abdominal. Observou-se que o rim direito era irrigado por duas artérias, de calibres diferentes, caracterizando uma duplicidade dessa artéria renal. A artéria secundária apresentava diâmetro 28,1\% menor que a principal do mesmo antímero e $34,3 \%$ menor que a principal do outro antímero. Ambas as artérias adentravam o rim pela

case of duplicity of the renal artery in a five year crossbred female weighting $18 \mathrm{~kg}$. The animal went to death due chronic hemoparasitosis and had latex injected by the thoracic aorta, on the sixth left intercostal space. It was dissected after being set in a $10 \%$ formaldehyde solution on the Animal Anatomy Laboratory of the Veterinary Medicine College, UNICASTELO, Fernandópolis, SP, aiming the visualization of the abdominal aorta branches. It was observed that the right kidney was irrigated by two arteries, of different diameters, characterizing duplicity of the renal artery. The secondary artery presented a diameter $28.1 \%$ smaller than the main artery of the same antimeter and $34.3 \%$ smaller than the main artery of the other antimeter. Both right arteries got into the kidney by the hilus region and presented practically parallel path. The left kidney presented irrigation by the corresponding renal artery, without any anatomical alteration in number. This kidney arterial duplicity should be considered in dogs, mainly when kidney alterations are present or when abdominal surgical interventions are driven near the kidneys, aiming to avoid errors due anatomical unfamiliarity of important structures.
\end{abstract} região do hilo renal e apresentavam trajetos praticamente paralelos. O rim esquerdo apresentava irrigação pela artéria renal correspondente, sem qualquer alteração anatômica em número. Esta duplicidade da artéria renal deve ser considerada em cães, principalmente quando alterações renais estiverem presentes ou quando intervenções cirúrgicas abdominais forem conduzidas próxima aos rins, visando-se, dessa forma, a evitar que erros sejam cometidos por desconhecimento anatômico de estruturas importantes.

Palavras-chave: duplicidade, artéria renal, cães.

\section{ABSTRACT}

Renal vessels abnormalities are noticed with low frequency in veterinary medicine ad may have direct implications on the regular functioning of these organs due blood flow change. Thus, the aim of this report is describe a
Key words: duplicity, renal artery, dogs.

As artérias renais transportam mais de $20 \%$ do sangue arterial da aorta abdominal (KÖNIG \& LIEBICH, 2004) e surgem, em carnívoros, assimetricamente das superfícies laterais desse vaso sangüíneo, ao nível da primeira vértebra lombar. A artéria renal direita é mais cranial que a esquerda, em conformidade com a posição relativamente mais cranial do rim direito (GOSHAL, 1986). Entretanto, de acordo com Koch, apud GOSHAL (1986), essas duas artérias podem surgir no mesmo nível. Às vezes, a artéria renal esquerda é dupla, surgindo separadamente da aorta

\footnotetext{
IDepartamento de Zootecnia, Universidade Estadual de Maringá (UEM), Faculdade de Medicina Veterinária. Av. Colombo, 5790, 87020-900, Maringá, PR, Brasil. E-mail: singaretti@ig.com.br. *Autor para correspondência.

IFFaculdade de Medicina Veterinária, Universidade Camilo Castelo Branco (UNICASTELO), Fernandópolis, SP, Brasil.
} 
abdominal, estando próximas uma da outra (GOSHAL, 1986).

Alterações arteriais renais são raras em medicina veterinária. A distribuição dessas artérias foi descrita em ovinos (MARDANI \& AHMADIGHOJABAIGLO, 2001), caprinos (ASLAN \& NAZLI, 2001), suínos e javalis (VODENICHAROV \& DANCHEV, 2003) e camelos (QIUSHENG \& YI, 2000), sem, no entanto, haver sido relatado qualquer tipo de duplicidade desses vasos sanguíneos.

Artérias renais acessórias foram descritas em 32 homens e três mulheres submetidos à cirurgia para reparo de aneurisma na aorta abdominal. O número médio de artérias renais acessórias observadas foi dois, podendo variar de um a quatro. Todos, exceto um paciente, apresentavam uma artéria renal acessória esquerda, e 23 deles uma artéria acessória direita (KARMACHARYA et al., 2006).

Assim, o objetivo deste artigo é descrever um caso de duplicidade da artéria renal direita em um cão, comparando-a com as descrições vasculares existentes.

Descreve-se um caso de duplicidade da artéria renal em um cão, fêmea, de cinco anos, sem raça definida e com $18 \mathrm{~kg}$. O animal veio a óbito após hemoparasitose crônica e foi submetido à injeção de látex pela aorta torácica, na altura do sexto espaço intercostal esquerdo. A peça anatômica foi dissecada após a sua fixação com solução de formaldeído a 10\%, no Laboratório de Anatomia Animal da Faculdade de Medicina Veterinária da UNICASTELO, Campus Fernandópolis, SP, visando-se à evidenciação das artérias renais.

Observou-se que o rim direito era irrigado por duas artérias, de calibres diferentes, caracterizando uma duplicidade dessa artéria renal. A artéria principal apresentava diâmetro de $1,47 \mathrm{~mm}$ e a secundária de $1,06 \mathrm{~mm}$, a qual situava-se caudalmente à primeira e à artéria renal esquerda, a qual apresentava diâmetro de 1,61mm e era mais caudal que a do antímero contralateral (Figura 1). As medidas foram obtidas com paquímetro digital $^{1}$.

Assim, a artéria secundária apresentava diâmetro 28,1\% menor que a principal do mesmo antímero e $34,3 \%$ menor que a principal do outro antímero. Ambas as artérias adentravam o rim pela região do hilo renal e apresentavam trajetos praticamente paralelos.

O rim esquerdo apresentava irrigação pela artéria renal correspondente, sem qualquer alteração anatômica.

No caso apresentado, a artéria renal direita apresentou-se mais cranial que a esquerda, como reportado por GOSHAL (1986) para os carnívoros. No entanto, diferiu do descrito por Koch, citado por GOSHAL (1986), o qual cita que as renais podem surgir no mesmo nível.

A duplicidade relatada foi observada na irrigação para o rim direito, diferindo do exposto por

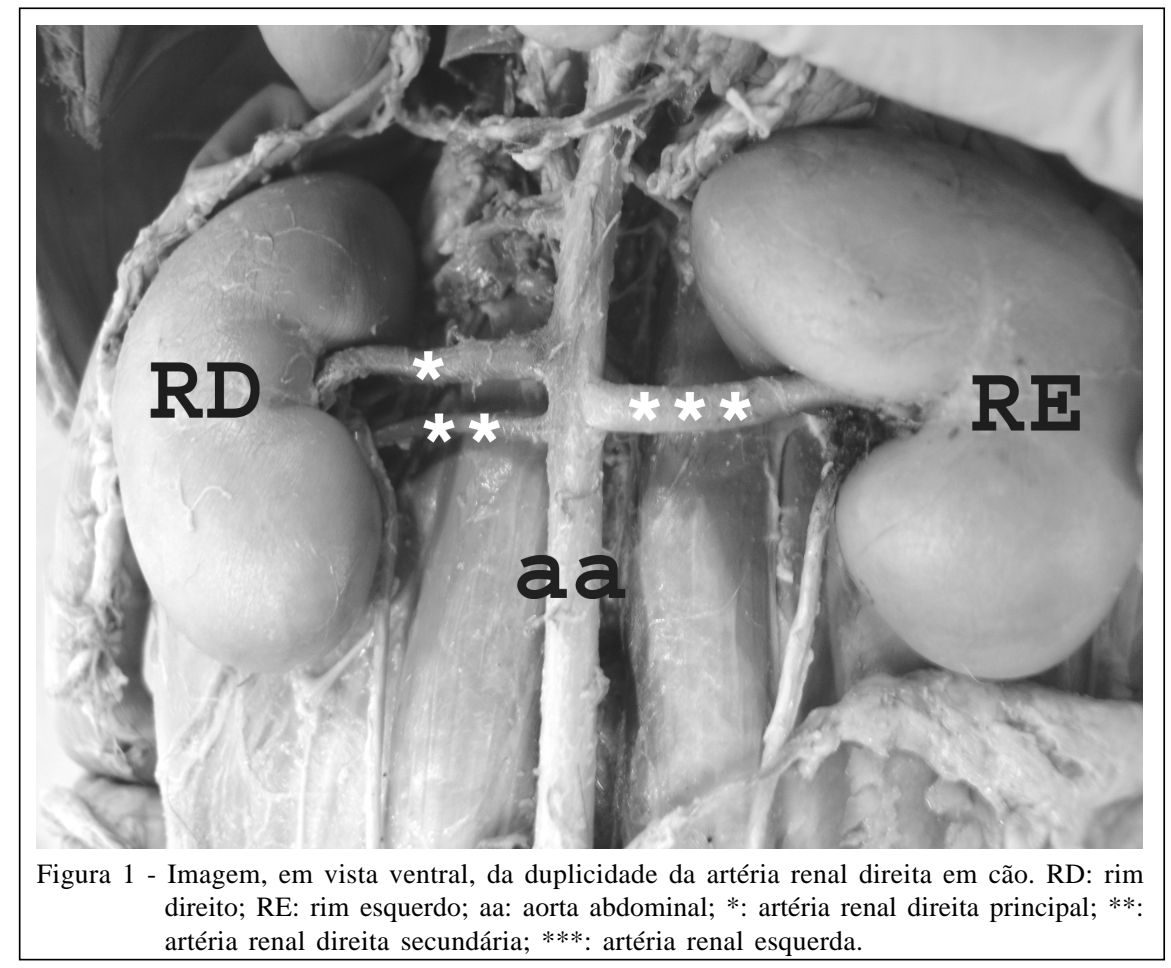

Ciência Rural, v.37, n.6, nov-dez, 2007. 
GOSHAL (1986) para os carnívoros, o qual afirma que esse tipo de alteração numérica vascular ocorre mais freqüentemente na referida artéria no antímero esquerdo. Em humanos (KARMACHARYA et al., 2006), as artérias renais acessórias foram mais freqüentes no rim esquerdo. Contudo, MARDANI \& AHMADI-GHOJABAIGLO (2001) em ovinos, ASLAN \& NAZLI (2001) em caprinos, VODENICHAROV \& DANCHEV (2003) em suínos e javalis, e QIUSHENG \& YI (2000) em camelos, descreveram apenas a distribuição desses vasos renais, sem relatar qualquer tipo de duplicidade.

Essa duplicidade arterial renal deve ser considerada em cães, principalmente quando alterações renais estiverem presentes ou quando intervenções cirúrgicas abdominais forem conduzidas próximas aos rins, visando-se, dessa forma, a evitar que erros sejam cometidos por desconhecimento anatômico de estruturas importantes.

\section{FONTE DE AQUISIÇÃO}

${ }^{1}$ Lee Tools Digital Caliper - China.

\section{REFERÊNCIAS}

ASLAN, K.; NAZLI, M. A comparative macro-anatomic investigation on the intrarenal segmentation of the renal artery in goats and Morkaraman sheep. Ind Vet J, v.78, n.2, p.139143, 2001

GOSHAL, N.G. Coração e artérias do carnívoro. In: GETTY, R. Anatomia dos animais domésticos de Sisson \& Grossman. Rio de Janeiro: Guanabara Koogan, 1986. Cap.55, p. 1536 .

KARMACHARYA, J. et al. Outcomes of accessory renal artery occlusion during endovascular aneurysm repair. J Vasc Surg, v.43, n.1, p. 8-12, 2006.

KÖNIG H.E.; LIEBICH, H-G. Órgãos urinários. In: Anatomia dos animais domésticos - texto e atlas colorido. Porto Alegre: Artmed, 2004. p.110.

MARDANI, K; AHMADI-GHOJABAIGLO, A. Branching pattern of the renal artery and urinary collecting system of the sheep kidney, using their cast by latex and mica. Iran J Vet Res, v.2, n.2, p.182-186, 2001.

QIUSHENG, C.; YI, L. Renal artery distribution in kidneys of bactran camels. Chin J Vet Sci, v.20, n.6, p.565-568, 2000.

VODENICHAROV, A; DANCHEV, S. Comparative anatomical study of renal artery architechtonics in the wild pig (Sus scrofa scrofa) and the domestic pig (Sus scrofa domestica). Bulg J Vet Med, v.6, n.1, p.1-8, 2003. 